\title{
Bienestar y felicidad: Un estudio de datos panel en el Municipio de Pasto, Colombia 2008 - 2010
}

\author{
Well-being and happiness: A study of panel data in the municipality of Pasto, Colombia during 2008-2010
}

Edinson Ortiz-Benavides 1

1 Mg. en Economía. Profesor Asistente Programa de Economía, Universidad de Nariño. Pasto, Colombia. e-mail: edinsoneconomia@gmail.com

\section{Resumen}

Introducción: La búsqueda de la felicidad es un fin de toda sociedad, para quien en ocasiones más riqueza, más renta y más bienes la conducen efectivamente a ella. Objetivo: Establecer la relación entre felicidad y el bienestar en términos subjetivos. Materiales y métodos: Se utilizó un modelo econométrico Logit Binomial, aplicado a los datos de panel provenientes de la encuesta previamente aplicada a 4.797 personas y 1.123 hogares en el municipio de Pasto, Colombia durante los años 2008 - 2010. Resultados: De manera significativa estadísticamente, las personas que manifiestan una mejor condición de bienestar se declaran un 13\% más feliz que aquellos que no lo hacen. Conclusión: El estudio permite concluir que este modelo encuentra la relación entre felicidad y bienestar en el municipio de Pasto, Colombia para el periodo de estudio. Se ratifica que las personas con mayor nivel de bienestar se declaran mayoritariamente felices, en contraste con aquellos que según la evaluación de su propia vida, no lo han sido.

Palabras clave: Felicidad; bienestar social; pobreza. (Fuente: DeCS, Bireme).

\begin{abstract}
Introduction: The pursuit of happiness is an end of society, where sometimes being wealthier, having more income and more goods lead effectively to it. Objective: To establish the relationship between happiness and welfare in subjective terms. Materials and methods: A Logit Binomial econometric model was applied to the Panel data from the survey previously applied to 4.797 individuals and 1,123 households in the municipality of Pasto, Colombia during the years 2008-2010. Results: Statistically significant, people who manifest a better condition of welfare state declared themselves to be $13 \%$ happier than those who do not. Conclusion: The study allows to conclude that this model finds the relationship between happiness and welfare in the municipality of Pasto, Colombia for the study period. It confirms that people with higher levels of welfare are declared to be happier than those who, according to their own evaluation, have not been.
\end{abstract}

Keywords: Happiness; welfare; poverty. (Source: DeCS, Bireme). 


\section{Introducción}

La felicidad ha representado una búsqueda tan desafiante como frustrante para la humanidad, si bien no existe consenso sobre su definición, debe tenerse en cuenta que no es un anhelo reciente. Muchos autores la asocian como un estado emocional que es producto de los logros alcanzados por una persona luego de una evaluación integral de su propia vida, que involucra desde reacciones fisiológicas como la sensación de bienestar y paz interior, hasta complejos contextos mentales que pueden involucrar emociones como la responsabilidad y el compromiso.

Ahora bien, por muchos años los economistas relacionaban ese concepto bello, que Aristóteles describía como el fin más preciado del hombre, en un concepto materialista, que asociaba la felicidad al 'tener', a la riqueza y los ingresos, asumiendo que el bienestar material (consumo) es una condición previa de la satisfacción subjetiva y que los cambios en la "felicidad" están directamente relacionados con los cambios en el poder adquisitivo. 1 Sin embargo, recientes investigaciones han planteado la necesidad de analizar la felicidad y sus determinantes desde una perspectiva más amplia, así como la incorporación de la misma a los principales fines de la política económica.

\section{El contexto: felicidad y bienestar en Pasto}

El municipio de Pasto capital del departamento, se caracteriza por tener una economía cíclica y volátil, sensible a choques, o perturbaciones, derivadas de las políticas económicas nacionales y regionales, y aún de las externas, dado el carácter de frontera que tiene el departamento de Nariño.2

No es menos importante la sensibilidad de la economía municipal a perturbaciones derivadas de fenómenos sociales como el conflicto armado y el narcotráfico, con sus secuelas de violencia y desplazamiento forzado, que ejercen presión en la demanda de soluciones, fundamentalmente, sobre los recursos públicos, que pueden propiciar un menor dinamismo de la economía local, que ha tenido coyunturalmente un notable crecimiento en el año $2006(6,43 \%)$ y uno menor en 2009 $(1,33 \%)$. Sin embargo, mantuvo un crecimiento promedio (simple) del 6\%, muy por encima del promedio departamental $(3,8 \%)$ y del nacional $(4,28 \%)$ en el período 2006 - 2010.3 Sin embargo, el aporte de esta economía al PIB nacional ha sido históricamente marginal. De conformidad con la disposición de datos a 2010, la participación de Pasto alcanzaba un $0,8 \%$ en el PIB nacional y un $53,83 \%$ en el PIB departamental.2

Esta dinámica productiva ha servido para que el municipio hoy ostente un nivel de bienestar por encima de los promedios departamental y nacional en indicadores como el de Necesidades Básicas Insatisfechas (NBI), Índice de Calidad de Vida (ICV) e Índice de Desarrollo Humano (IDH), llevándolo a tener una mayor proporción de su población declarada como feliz $(81,92 \%)$ frente al $80,02 \%$ del promedio departamental y el $77 \%$ para el promedio nacional en el año 2012.

En las últimas mediciones internacionales sobre felicidad se ha ubicado a Colombia como uno de los países más felices del mundo, y por consiguiente Pasto resulta como una de las comunidades más felices a nivel mundial, aunque su PIB per cápita similar al de Colombia, sea entre 5 y 15 veces menor al de las principales economías mundiales como Noruega, Luxemburgo y Estados Unidos. (Tabla 1).

Tabla 1. Indicadores de bienestar y felicidad en Pasto 2012

\begin{tabular}{|c|c|c|c|}
\hline Indicador & Pasto & Nariño & Colombia \\
\hline NBI 2012 & 16,2 & 43,79 & 27,77 \\
\hline ICV 2012 & 84,04 & 69,3 & 78,8 \\
\hline IDH 2012 & 0,89 & 0,773 & 0,78 \\
\hline \% Feliz 2012 & 81,92 & 80,02 & 77 \\
\hline $\begin{array}{l}\text { PIB per cápita } 2010 \text { (\$ de } \\
\text { 2005) }\end{array}$ & $8.308,7$ & $3.816,1$ & $9.340,1$ \\
\hline Línea de pobreza 2010 & 43,2 & 56,14 & 37,2 \\
\hline
\end{tabular}




\section{Felicidad y economía}

Las investigaciones empíricas sobre felicidad empiezan alrededor de 1960. En el campo de la sociología, la felicidad fue el principal indicador para evaluar la calidad de vida de la población.4,5 Este campo de investigación también fue preocupación de la psicología y de la medicina.6-9

Gracias a los estudios de Bemard Van Praag,10 Richard Easterlin,11 y Tibor Scitovsky12 se atribuye el hecho de que el interés por la felicidad retorne al ámbito de la ciencia económica. Más tarde, economistas como Layard,13 Veenhoven,14 Oswald $_{15}$ y Frey and Stutzer 16 han continuado con esta investigación. Finalmente, a partir de los trabajos de Kahneman,17 se instaura la investigación sobre la Economía de la Felicidad como una nueva subdisciplina de la Economía del Bienestar.

Los resultados de los estudios empíricos de Easterlin $_{11}$ y Scitovsky,12 utilizando datos proporcionados por las personas acerca de su propia felicidad o "bienestar subjetivo", condujeron a que se empezara a poner en duda la relevancia de la riqueza como variable explicativa primordial del bienestar.

Poco después de la publicación de estas obras dos economistas, también americanos, se propusieron estudiar la satisfacción en el trabajo con vistas a explicar el comportamiento del mercado de trabajo.18,19 En los países Bajos (Escuela de Leyden) Bernard Van Praag 10 lleva a cabo estudios sobre desigualdad y la percepción que de esta hacen los individuos y en la London School of Economics, Richard Layard ${ }_{13}$ estudia las consecuencias que los resultados de estos estudios empíricos pueden tener en lo concerniente a las recomendaciones en materia de política económica que de ellos puedan derivarse.10,13 Ya en los años 90 los trabajos de Blanchflower, Clark y Oswald, entre otros, han contribuido a que este tipo de estudios se hayan extendido y se valoren en el ámbito de la economía.20

\section{Felicidad y bienestar}

En términos conceptuales, tanto en Psicología como en Economía, así como en otras Ciencias
Sociales, hay fundamentalmente dos aproximaciones al tema de la felicidad y una cierta tensión entre ellas. La primera aproximación tiene como referencia a Bentham y es de tipo subjetivo - hedonista - individualista; la segunda cuyo referente principal es Aristóteles la cual es una aproximación de tipo objetivo - eudamonista - relacional. Sin duda, la primera aproximación cuenta con la aprobación de muchos economistas y es, con diferencia, la más extendida mientras que resulta bastante difícil encontrar en la literatura económica que trata sobre el tema, economistas que se refieran a la felicidad en el sentido eudamonista - relacional.20

Como señalan Bruni y Porta: "cuando hoy los economistas usan el término felicidad conscientemente o no - están en línea con Bentham y lejos de Aristóteles".21 La aproximación eudamonista es, pues, todavía marginal en economía y cabe decir que, debido al énfasis que pone en las relaciones sociales así como en el desarrollo integral de la persona, suele considerarse, a veces, en línea con la aproximación de las capacidades (capability approach) de Amartya Sen aunque éste niega, según Bruni y Porta,21 la inspiración Aristotélica.20

Según Aristóteles, la felicidad crece en función de cómo la gente interactúa dentro de la sociedad y cómo desarrolla sus potencialidades.21 Por tanto, ese filósofo le da un valor especial al compromiso de participar en la vida civil o política, sin la cual la vida humana no puede florecer.22

Para Argyle,23 la felicidad se define como un estado emocional que es producto de los logros alcanzados por una persona, que involucra desde reacciones fisiológicas como la sensación de bienestar y paz interior, hasta complejos contextos mentales que pueden involucrar emociones como la responsabilidad y el compromiso.24

En términos más concretos, pueden existir varias formas de entender la felicidad:

(a). "se puede decir que se está feliz porque ha ocurrido algo particular (mi equipo ganó la copa 
América) y eso me deja satisfecho o más satisfecho de lo que estaba antes; (b). la gente puede estar sintiéndose feliz ahora, durante un determinado intervalo de tiempo independientemente de tener o no motivos conscientes y definidos para la sensación de alegría, y (c) puedo decir que soy' feliz porque al evaluar mi vida en su conjunto siento que, en general, puedo considerarme satisfecho con ella".25

En el último caso, la felicidad no sólo es una sensación local (estar feliz), sino que incluye una reflexión, o sea que la felicidad es un sentimiento que proviene de una evaluación global de la vida (ser feliz).22 La felicidad que interesa en esta investigación se refiere sobre todo, al ser feliz antes que al estar feliz.

En este sentido, no es fácil "construir" una concepción de la felicidad que tenga el vigor de una categoría científica, pues existen muy diversas formas de acercarse a ese sentimiento o sensación. Lo primero que resalta es que la felicidad es una percepción muy individual, una "opción individual" y, por tanto, una apreciación muy subjetiva de la vida. No depende tanto del entorno -aunque no puede prescindirse de él--, cuanto de aquello a lo que cada persona da valor o corresponde a lo que le interesa o aspira.26

De acuerdo con la teoría económica ortodoxa se parte de la creencia de que mayores niveles de riqueza y de renta configuraban una realidad social con unas condiciones de vida mejores (mayor consumo), mayor bienestar $\mathrm{y}$, por extensión, mayor felicidad. Dicho de otra manera, la ciencia económica tendía a asumir que el bienestar material (consumo) es una condición previa de la satisfacción subjetiva y que los cambios en la "felicidad" están directamente relacionados con los cambios en el poder adquisitivo. ${ }_{27}$ Siguiendo este postulado, la política económica y social ha tenido como objetivo un crecimiento económico (crecimiento del PIB percápita) que eleve el ingreso de los individuos y por ende su nivel de bienestar.

Esa visión ha dominado el análisis económico del tema, no obstante nuevas corrientes de académicos no comparten tal perspectiva, dando origen a trabajos teóricos subjetivistas al respecto. Estos trabajos presentan un alto grado de escepticismo sobre si la utilidad puede ser generalmente derivada de las elecciones de los individuos, proponiendo una aproximación subjetiva mediante la recolección de información de los hogares, proveniente de las encuestas que ofrecen una posibilidad más amplia de entender el comportamiento humano tanto desde lo teórico como de lo empírico.28

El concepto de satisfacción subjetiva revelada, asociada a la felicidad individual incluye tanto la utilidad experimental como procedimental, tal definición admite que cada individuo tiene sus propias ideas acerca de la felicidad y que el comportamiento observado es un indicador incompleto para reconocer el bienestar individual. Aceptando esta visión, la felicidad individual puede ser capturada y analizada por medio de encuestas, en las que se pregunta qué tan satisfactoria es su vida, reconociendo de manera implícita al individuo como la fuente más acertada de información sobre la calidad de su propia vida. 29

Así como puede decirse que la felicidad es algo subjetivo a nivel individual, también es una cuestión objetiva a nivel social o colectiva, pues corresponde a una de las más milenarias aspiraciones de la humanidad.26 A este respecto, la felicidad colectiva puede ser capturada y analizada como la sumatoria de comodidades individuales recogidas en un indicador sintético como el Índice Internacional de Felicidad (Happy Planet Index - hpi) un estudio realizado por la New Economics Fundation (NEF) en 2012.30

Conforme lo anterior, redefinido el papel de la economía en el estudio de la felicidad, este concepto aún con algunas diferencias, se equipará mucho a los de bienestar y calidad de vida, pues se demostrará que guardan correlaciones positivas fuertes, tanto a nivel individual $\mathrm{y}$ colectivo que no dan pie a existencia de paradojas, como si sucede cuando se la relaciona con el bienestar material o riqueza (medida por ingresos). Así como en Ramírez 22 se empezará por decir que la palabra bien-estar es un concepto que en el idioma español tiene una limitante, dado que 
en inglés (well-being) el verbo "to be" significa "ser y estar" y en su traducción sólo se toma en cuenta el "estar" de las personas y no el "ser" de las mismas. En este sentido, en este artículo se propone que frente al concepto de bienestar se use también el del buen vivir de manera indistinta.

El concepto de bienestar es un constructo abstracto con connotaciones subjetivas, pero que está correlacionado con factores económicos objetivos. Así, el bienestar individual alude a la conciencia que tiene cada individuo de haber cubierto sus necesidades privadas, y que se puede capturar con la manifestación expresa de los individuos de su condición de "no pobre", concepto asociado al de pobreza subjetiva, que como su nombre lo indica se basa en las apreciaciones que tienen los individuos acerca de sus condiciones de vida y del entorno que los rodea.

Dentro de esta percepción general se distinguen varias dimensiones del bienestar subjetivo: bienestar económico, bienestar psicológico y bienestar social. En cada una de estas vertientes se resaltaría la valoración que el individuo realiza de su situación económica, de los estados afectivo-emocionales y psicológicos o de funcionamiento social respectivamente. En esta investigación la pregunta puntual que se utiliza es: ¿Usted se considera pobre?, y tomamos aquella respuesta donde el individuo no se ubica en tal situación (no pobre).

Por su parte, el bienestar social o colectivo, estaría originado por el hecho de que un elevado porcentaje de los individuos, que se integran en un determinado grupo social, satisfagan sus necesidades. Éste por su parte se puede capturar de una manera más objetiva a través de un indicador sintético como el Índice de Desarrollo Humano calculado desde 1990 por el Programa de las Naciones Unidas para el Desarrollo (PNUD) basado en los trabajos de Amartya Sen. Se trata de un indicador social estadístico que toma en cuenta tres parámetros: a) vida larga y saludable (medida según la esperanza de vida al nacer) b) educación (medida por la tasa de alfabetización de adultos y la tasa bruta combinada de matriculación en educación primaria, secundaria y terciaria) y c) nivel de vida digno (medido por el PIB per cápita en USD).27

Por otro lado, según la OMS la calidad de vida alude a "la percepción que un individuo tiene de su lugar en la existencia, en el contexto de la cultura y del sistema de valores en los que vive y en relación a sus objetivos y expectativas, sus normas y sus inquietudes. Se trata de un concepto muy amplio que está influido por la salud física del sujeto, su estado psicológico, su nivel de independencia, sus relaciones sociales, así como su relación con los elementos esenciales de su entorno".31 Ahora bien, si desgranamos la definición previa debemos distinguir dentro del concepto de calidad de vida dos vertientes: la presencia de las condiciones consideradas necesarias para una vida buena y la práctica del vivir en cuanto tal.

La calidad de vida a escala social sólo puede entenderse bajo el prisma del primero de los significados. En cambio, si la aplicamos a escala individual el término puede usarse en ambas acepciones: disfrutar de las condiciones adecuadas en una vida realizada. Esto nos lleva directamente a las valoraciones individuales sobre las condiciones personales de vida como indicador social, esto es, el problema del bienestar subjetivo y la felicidad. Este concepto se puede capturar al interior de países con indicadores como por ejemplo el Índice de Calidad de Vida para el caso de Colombia, el cual resulta un indicador objetivo.

\section{Felicidad e ingresos}

El ingreso es una variable esencial en la vida de los hombres. El ingreso posibilita una buena alimentación, contar con un buena atención en salud, una buena educación, tener una vivienda en condiciones, y acceder a un conjunto de factores elementales para disponer de una calidad de vida que permita a la sociedad ubicarse en un punto óptimo de bienestar. ¿Pero el ingreso hace más feliz a los hombres? Si el ingreso mejora ¿es de esperar que las personas sean más felices? ¿Cómo influyen los ingresos de los demás en la felicidad de cada uno? Este tipo de preguntas guían los trabajos modernos sobre economía y felicidad. Y los resultados, lejos de ser contundentes, son 
variados, contradictorios y en algunos casos ambiguos. Pero todos ellos tienen un punto en común: en mayor o menor medida, arrojan evidencia que la economía y la felicidad tienen planos de unión.1 Investigaciones empíricas de los últimos cincuenta años en los contextos colombiano, latinoamericano y mundial, señalan que en un mismo país y tiempo determinado, los individuos con más ingresos son más felices, pero los incrementos en ese nivel de ingresos son acompañados cada vez de menores incrementos en los niveles de felicidad subjetiva revelada, hasta un punto en el cual los incrementos de ingresos pueden generar pérdida de felicidad. Este comportamiento marginal decreciente de dicha relación ingresos - felicidad es lo que se conoce en la teoría económica como la Paradoja de Easterlin, por los trabajos que este autor viene haciendo con datos de la economía norteamericana desde 1974.

Sorprendentemente cuando se hace el estudio comparativo entre países, los países ricos tienden a ser más felices que los países pobres e incrementos en su ingreso per cápita muestran el mismo comportamiento marginal decreciente respecto al índice de felicidad global del país, aunque con menor significancia estadística, debido a la agregación macroeconómica de los datos. Este hecho paradójico da pie para que algunas personas o países pobres puedan en determinado momento ser más felices que aquellos más afortunados. Las mismas investigaciones señalan US\$75.000 dólares anuales para individuos y US $\$ 20.000$ dólares per cápita para los países, los umbrales de ingresos a partir de los cuales incrementos de éste generan desutilidad o pérdida de felicidad.

Esta constatación sugiere que, una vez alcanzada la satisfacción material básica, las relaciones entre ingreso y felicidad se tornan notablemente más complejas. La paradoja de Easterlin ${ }_{11}$ alude al hecho de que en las sociedades desarrolladas las elevaciones de los niveles de renta no generan un incremento en los niveles generales de bienestar del colectivo, aunque persiste una relación directa entre niveles de bienestar individual e ingreso personal. Es decir, lo que es válido para el individuo no lo sería para la sociedad considerada en su conjunto. Para explicar esta aparente paradoja se han adelantado una serie de hipótesis, entre las cuales destacan las ligadas a la renta relativa en sus dos versiones: comparaciones sociales y adaptación.32 A estas hipótesis cabría añadir las explicaciones que hacen especial hincapié en aspectos de orden axiológico o valorativo, psicológico y sociológico, los cuales también deben ser tenidos en cuenta a la hora de analizar la complejidad de este hecho.

Las primeras explicaciones al tema fueron formuladas por el propio Easterlin,11 quien explicaba la paradoja basándose en la teoría del ingreso relativo de Duesenberry.33 Según esta teoría la satisfacción que una persona obtiene de su nivel de ingresos (consumo) no depende del nivel absoluto de los mismos sino del nivel relativo; de manera que la satisfacción o bienestar subjetivo de una persona está positivamente relacionado con su nivel de ingresos y negativamente con los ingresos de los demás.

Si sus ingresos (consumo) aumentaran pero menos que los de los demás su bienestar subjetivo disminuiría. Asimismo, en un contexto extremo en que sólo importen los ingresos relativos, si en el curso del crecimiento económico aumentaran los ingresos absolutos de todos los individuos en la misma medida esto no implicaría mayores niveles de felicidad porque los efectos positivos de tener mayores ingresos se contrarrestarían con los negativos relacionados con el aumento de los ingresos de los demás. Este fenómeno se conoce también como efecto marea.34 pues actúa de modo semejante a una marea creciente que hace elevar simultáneamente a todos los camarotes de un barco pero que, obviamente, mantiene la diferencia entre las distintas clases".20

Si ligamos esta explicación con la idea de la noria hedónica (hedonic treadmill) o el binomio logrosaspiraciones surge la hipótesis de la Adaptación Hedónica (o teoría del set point), según la cual la felicidad de un individuo dependerá fundamentalmente de sus ingresos en relación a sus aspiraciones y estas, a su vez, dependen de los ingresos medios de las personas de su entorno. 35 Así, a medida que aumentan los logros del 
individuo también lo hacen sus aspiraciones, con lo cual el nivel de satisfacción se mantendría inalterado.

En otras palabras, esta teoría sostiene que cada individuo tiene unas características más o menos innatas (congénitas) y que en función de estas características queda vinculado a un determinado nivel de felicidad que apenas cambia a lo largo de toda su vida. Así cuando el nivel de renta aumenta y con ello la cantidad y calidad de bienes que se pueden obtener se produce un incremento de bienestar subjetivo durante un tiempo para (una vez adaptados a la nueva situación) volver al nivel de satisfacción de referencia (set point). Easterlin llega a esta conclusión tras hacer un seguimiento en el tiempo a grupos de personas (cohortes) que eran estudiadas durante todo el ciclo de su vida. En palabras de este mismo autor: "La relación entre felicidad e ingreso es muy compleja. En un momento cronológico dado, los que poseen mayores ingresos son en promedio más felices que los que ganan menos. Pero si se considera el ciclo de vida en su conjunto, la felicidad media de un grupo permanece constante, aunque exista un incremento notable de ingresos" .36

En suma, los sujetos no dejan de percibir que, a causa de la adaptación hedónica y de la pugna social, las aspiraciones se modifican en función de las circunstancias efectivas de cada momento. Por consiguiente, se dedica una cantidad de tiempo desproporcionada a la obtención de objetivos monetarios, a expensas de otros bienes de carácter no material (por ejemplo, la vida familiar y la salud) disminuyendo el bienestar subjetivo respecto del nivel esperado. Los "otros bienes" no ligados estrictamente al bienestar material pueden estar desempeñando una función mayor de la esperada, con lo cual, un cambio de asignación del tiempo que diera preeminencia a la vida familiar y a la salud aumentaría el bienestar subjetivo.27

Pero independiente de las explicaciones económicas, psicológicas y sociológicas a la relación paradójica entre felicidad e ingresos, lo que se evidencia es la necesidad de estudiar la felicidad y sus determinantes desde una perspectiva más amplia que la que ha sido habitual en el dominio de la ciencia económica. Aspectos como el acceso a un trabajo, el estado civil, la salud y el nivel educativo son algunos de los generalmente citados como principales determinantes de la felicidad, y su importancia diferiría según la edad, el género y el lugar de residencia.11

En este sentido, la literatura teórica y la evidencia empírica sobre el tema (Frey y Stutzer;16 Layard;37,38 Veenhoven ${ }_{14,39,40}$ Easterlin;11 Frey y Stutzer $_{28}$ ) ha identificado relaciones importantes entre ellas. En particular, la investigación de Cruz y Torres 29 para el caso colombiano, encuentra que la salud tiene una relación positiva frente a la percepción de satisfacción o felicidad, encontrándose que aquellos que gozan de buena salud tienen un $87 \%$ más de probabilidad de responder que sus condiciones de vida son superiores en comparación a los que se encuentran enfermos. Evidencia que agrega un elemento más para explicar la percepción de bienestar o felicidad por parte de los colombianos, una buena salud, aparte de estar en un estado físico aceptable, también permite el desempeño de una actividad productiva, entre otros beneficios.

El estudio sobre satisfacción subjetiva de los colombianos, los jefes de hogar en Colombia que no se consideran pobres tienen 2,3 veces más de probabilidad de responder que sus condiciones de vida son satisfactorias o que son felices frente a los que se consideran pobres; los desempleados tienen $17 \%$ menos probabilidad de tener buenas condiciones de vida, que si estuvieran empleados. El hecho de ser desempleado disminuye las probabilidades de responder que sus condiciones son buenas o muy buenas en $4 \%$ y $0,5 \%$, respectivamente. 41

De acuerdo con las estimaciones, el género del individuo influye en la percepción de satisfacción o felicidad de los colombianos a favor del género masculino, con un $7 \%$ más de probabilidad de responder que sus condiciones de vida son mejores. Resultado que debe ser visto con cautela, debido al tipo de sujetos encuestados. En el caso que el jefe sea mujer, supone que ella se encuentra asumiendo la responsabilidad del manejo del 
hogar y que puede estar sin pareja, lo cual se ratifica al observar la composición de la muestra, donde el $72 \%$ de los solteros son mujeres jefe de hogar. El efecto marginal de ser hombre en Colombia sobre tener buenas y muy buenas condiciones de vida aumenta en 1,5\% y 0,2\%, respectivamente. 41

Las variables edad y edad al cuadrado tiene signos negativo y positivo, respectivamente, similar a lo encontrado en la literatura internacional. Como resultado se tiene, que la percepción de satisfacción en Colombia tiene forma de U con respecto a la edad, donde su punto mínimo se encuentra alrededor de los 50 años.

El mismo estudio, respecto a la educación, indica que ésta juega un papel significativo en la percepción de satisfacción o felicidad. Por cada año más de educación, la probabilidad de responder que sus condiciones de vida son malas disminuye en $0,07 \%$ y regulares en $0,5 \%$. Mientras que aumenta la probabilidad de contestar que sus condiciones son buenas y muy buenas. La educación no sólo es el medio por el cual los individuos pueden acceder a mayores ingresos, sino que es uno de los medios que permite obtener un mayor grado de satisfacción o felicidad.41

Conforme a lo anterior, ha quedado en evidencia la necesidad de vincular como determinantes de la felicidad, no solo el ingreso, sino una medida de bienestar más integral (Bienestar Subjetivo Revelado-BSR) que en asocio con indicadores de salud, situación laboral, educación, edad y estado civil, contribuyan a una mejor explicación de este anhelo global.

A nivel individual todos estos aspectos sobre BSR son tenidos en cuenta en la valoración subjetiva de las personas cuando se declaran "no pobres", pues de acuerdo con la literatura, la percepción subjetiva de la pobreza no necesariamente está sujeta al ingreso del hogar, porque los individuos no sólo valoran los niveles absolutos del ingreso, sino que también se interesan en su posición relativa frente a su grupo social de referencia.29 Por su parte, a nivel colectivo o agregado buena parte de estos aspectos se vinculan en el Índice de
Desarrollo Humano (IDH), por tanto, ambas medidas de bienestar o buen vivir: "no pobre" e IDH, son buenos regresores a la hora de explicar la felicidad, sin el riesgo de que se presenten hechos paradójicos.

\section{Materiales y métodos}

La función de felicidad se estima mediante un Logit Binomial, debido a que la variable dependiente es una variable discreta binaria, expresado mediante la siguiente forma funcional:

$$
\begin{aligned}
& F=\operatorname{Pr}\left(F_{i, t}=1 / X_{i, t}\right)=G\left(Z_{i, t}\right)=\frac{e^{Z_{i, t}}}{1+e^{Z_{i, t}}} \\
& F=\operatorname{Pr}\left(F_{i, t}=1 / X_{i, t}\right)=G\left(\beta_{0}+\beta_{1} B S R_{i, t}\right. \\
& \left.\beta_{2} X_{2, t}+\cdots+\beta_{k} X_{k, t}+\mu_{i, t}\right)
\end{aligned}
$$

Donde:

F: Función de felicidad

$F_{i, t}$ : Felicidad subjetiva revelada por el individuo $\boldsymbol{i}$, en el periodo $\boldsymbol{t}$.

$\operatorname{Pr}\left(F_{i, t}=1 / X_{i, t}\right): \quad$ Probabilidad de que el individuo $\boldsymbol{i}$, se declare "feliz" en el periodo $\boldsymbol{t}$.

$X_{i, t}=$ Vector de variables independientes explicativas correspondientes al individuo $\boldsymbol{i}$, en el periodo $\boldsymbol{t}$. Aquí se encuentran la variable de interés como medida del bienestar (BSR) y las demás explicativas como edad, educación, ingresos, estado civil, etc.

$\mathrm{G}=$ Función logística

$Z_{i, t}=\beta_{0}+\beta_{1} B S R_{i, t}+\beta_{2} X_{2, t}+\cdots+\beta_{k} X_{k, t}+\mu_{i, t}$ $\beta_{0}=$ Intercepto

$\beta_{1}, \beta_{2} \ldots \beta_{k}=$ Coeficientes de variables independientes

$B S R_{i, t}=$ Bienestar subjetivo revelado por el individuo $\boldsymbol{i}$, en el periodo $\boldsymbol{t}$. Corresponde a la respuesta del individuo como "no pobre".

$\mu$ : es el término de perturbación aleatoria

$\boldsymbol{i}, \boldsymbol{t}$ : Representa los subíndices para el individuo $\boldsymbol{i}$, en el tiempo o periodo $t$.

Respecto a los datos, para el nivel macroeconómico, el modelo empírico utilizó información a nivel de países, provenientes de la Fundación New Economics, el PNUD y el Banco Mundial en relación con el HPI, IDH y PIB per cápita respectivamente calculados para el año 
2012. A nivel microeconómico los datos proviene del estudio panel mediante encuesta aplicada a 4.797 individuos (vinculados a 1.123 hogares) en el municipio de Pasto, Colombia durante los años 2008 - 2010 en el marco de la investigación Coyuntura Social de los Hogares en Pasto financiada por la Universidad de Nariño. La encuesta contó con un nivel de confianza del 95\% y un error del 5\%, recogió de manera aleatoria estratificada información de individuos de todos los estratos sociales, áreas geográficas, nivel de estudios y de salarios del casco urbano municipal, por lo tanto se considera que la muestra es representativa de la población ocupada y no ocupada de esta localidad.

Se advierte que en principio el número de años del panel puede resultar limitado al ofrecer pocos grados de libertad, ante lo cual se debe ser precavido en las inferencias.

\section{Resultados y discusión}

Los datos indican una alta variabilidad sobre todo en el PIB per cápita, ingreso laboral y la educación, evidenciando heterogeneidad de los mismos. El promedio general del PIB es de USD $\$ 12.771,6$ con una desviación de USD\$14.624,3 tomando valores entre USD\$ 319 y USD\$ 87.478. El promedio general de ingresos es de $\$ 826.458,7$ con una desviación de $\$ 889.152,5$, tomando valores entre $\$ 0$ y $\$ 8.000 .000$. Así mismo, el promedio de educación en la población es de 9,6 años con una desviación de 4,7 años, tomando valores entre 0 y 18 años de educación.

Tabla 2. Variables macroeconómicas de países del mundo y microeconómicas individuos de Pasto

\begin{tabular}{|c|c|c|c|c|c|c|}
\hline \multicolumn{7}{|c|}{ Variables macroeconómicas - Países del mundo } \\
\hline Variables & Definición & Obs. & Media & Desviación & Mínimo & Máximo \\
\hline hpi 2012 & Índice Internacional de Felicidad & 151 & 42,23821 & 9,111702 & 22,59 & 64,04 \\
\hline PIBper 2012 & PIB Per cápita (USD\$ de 2005) & 187 & 12770,56 & 14624,34 & 319 & 87478 \\
\hline IDH 2012 & Índice de Desarrollo Humano & 187 & 0,6755615 & 0,1710093 & 0,3 & 0,96 \\
\hline \multicolumn{7}{|c|}{ Variables microeconómicas - Individuos de Pasto } \\
\hline Variables & Definición & Obs. & Media & Desviación & Mínimo & Máximo \\
\hline Feliz & Dummy de felicidad & 2134 & 0,8894096 & 0,3136978 & 0 & 1 \\
\hline Nopobre & Dummy de no pobre & 4797 & 0,2614134 & 0,4394504 & 0 & 1 \\
\hline Desocupado & Dummy de desempleado & 4797 & 0,1669794 & 0,3729963 & 0 & 1 \\
\hline Ingresoslab & Ingresos laborales ( $\$$ corrientes) & 1626 & 826458,7 & 889152,5 & 0 & 8000000 \\
\hline Casado & Dummy de casado & 4797 & 0,358349 & 0,4795654 & 0 & 1 \\
\hline Educación & Años de educación & 4224 & 9,616477 & 4,710534 & 0 & 18 \\
\hline Femenino & Dummy de mujer & 4797 & 0,561601 & 0,4962425 & 0 & 1 \\
\hline Edad & Edad en años & 4600 & 33,92407 & 21,06204 & 0,01 & 95 \\
\hline Mestizo & Dummy de mestizo & 4797 & 0,8684595 & 0,3380258 & 0 & 1 \\
\hline
\end{tabular}

Los datos ratifican a Colombia como uno de los países más felices del mundo, ocupando el tercer puesto después de Costa Rica y Vietnam entre 151 países evaluados, pero ocupa el puesto 85 en cuanto a PIB percápita con usd\$ 8.711 y el puesto
91 en cuanto a IDH con un indicador de 0,72 entre 187 países evaluados.

El PIB per cápita del país es inferior al promedio mundial en usd\$4.060, pero su promedio de IDH es mayor al promedio mundial en 0,04 puntos. 
Mediante análisis cruzado de variables, se encuentra a nivel macroeconómico que los países con un PIB per cápita inferior a usd\$ 10.000 (51\% de la muestra) son más felices que aquellos que poseen un PIB superior. Así mismo países con un IDH superior a 0,5 puntos $(79,14 \%$ de la muestra) son más felices que aquellos que poseen un IDH inferior.

A nivel microeconómico se observa que las personas que se declaran como no pobres son más felices en 14 puntos porcentuales que aquellos que sí lo hacen. Así mismo, los desocupados, hombres, negros e indígenas, casados y las personas con primaria o menos años de estudios, son más felices que sus contrapartes con mínimas diferencias. (Tabla 3).

Tabla 3. Felicidad en Pasto según grupos de individuos

\begin{tabular}{lrr}
\hline \multicolumn{1}{c}{ Grupos de individuos } & \% Feliz & \% No Feliz \\
\hline No pobres & 94,6 & 5,4 \\
Pobres & 80,8 & 19,2 \\
Desocupados & 90 & 10 \\
Ocupados & 88,8 & 11,2 \\
Mujeres & 88,8 & 11,2 \\
Hombres & 89,2 & 10,8 \\
Mestizos & 88,9 & 11,1 \\
Negros e Indígenas & 89,4 & 10,6 \\
Casados & 89,8 & 10,2 \\
No Casados & 88,5 & 11,5 \\
Personas con primaria o menos & 89,4 & 10,6 \\
Personas con más de 5 años de & & \\
estudios & 88,8 & 11,2 \\
\hline
\end{tabular}

A nivel macroeconómico las regresiones econométricas simples de Mínimos Cuadrados
Ordinarios (MCO) debido a la disponibilidad de datos, muestran una relación positiva entre la felicidad expresada en los países (HPI) y su índice de desarrollo humano (IDH). Es decir, por cada punto porcentual que aumente el IDH, la felicidad lo hace en 15,6 puntos aproximadamente. Este resultado es robusto a diferentes controles. Así mismo, la relación entre felicidad y PIB per cápita se muestra marginal decreciente, ratificando la existencia de la Paradoja de Easterlin con un punto de inflexión en USD $\$ 21.815,6$ a partir de los cuales, incrementos de ingresos en los países generan una reducción del nivel de felicidad de los mismos. (Gráficos 1 y 2).

A nivel microeconómico utilizando el modelo empírico descrito, las pruebas econométricas (Breusch Pagan) evidenciaron con un 99\% de confianza, la no presencia de heterogeneidad no observada en el término de error, lo cual implica que se puede utilizar Mínimos Cuadrados Agrupados (MCA) en las regresiones logísticas.

Gráfico1. Relación felicidad v.s. IDH - Pasto, 2012

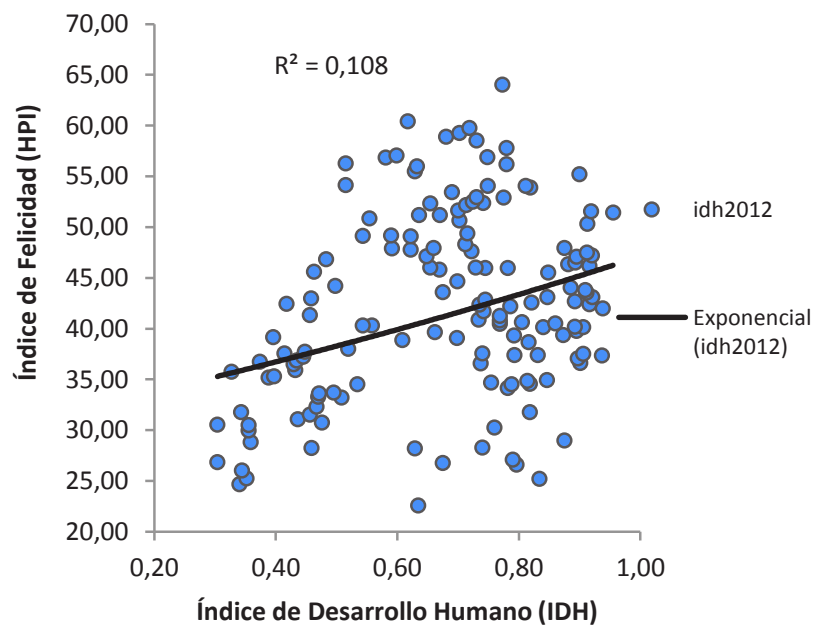


Gráfico 2. Relación felicidad v.s. PIB per cápita - Pasto, 2012

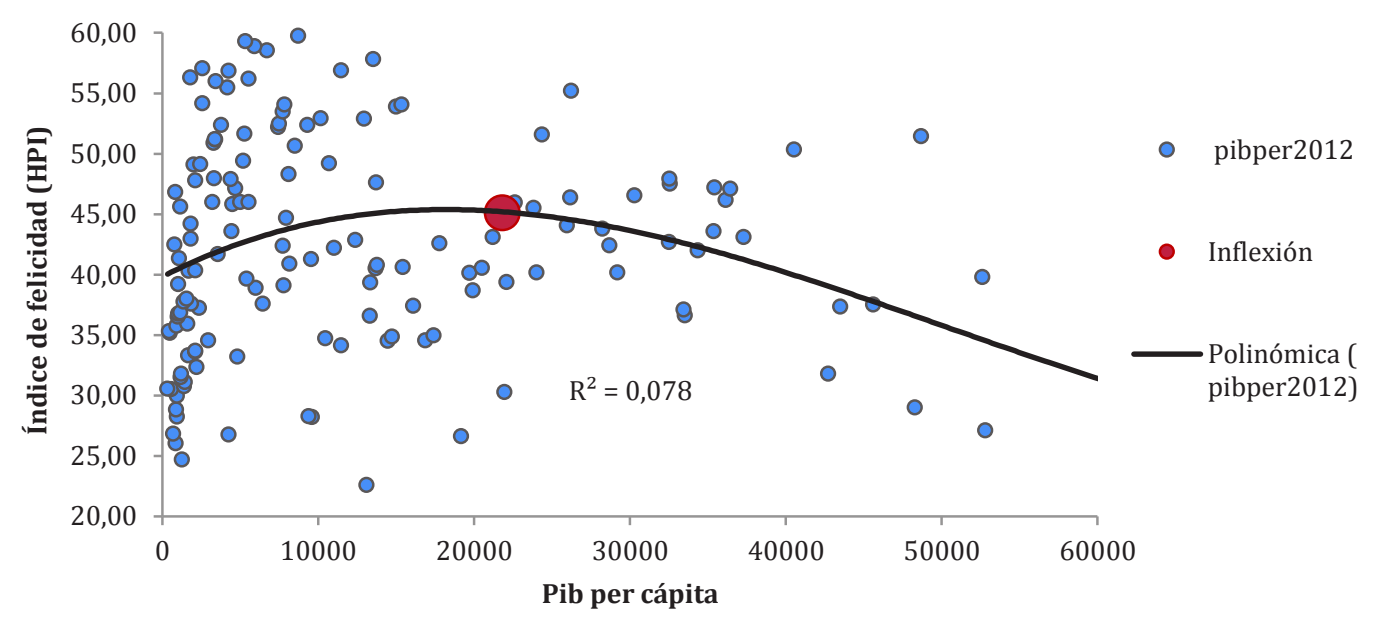

Los datos corroboran la relación positiva entre el bienestar (BSR) y la felicidad expresada por los habitantes de Pasto. Estos resultados se obtienen a un nivel del 99\% de confianza y resultan persistentes a diferentes controles. (Tabla 4).

Tabla 4. Bienestar y felicidad en Pasto, 2008 - 2010

\begin{tabular}{|c|c|c|c|c|c|}
\hline \multirow{2}{*}{ Variables } & \multicolumn{5}{|c|}{ Felicidad - Modelo Logístico } \\
\hline & (1) & $(2)$ & (3) & (4) & (5) \\
\hline \multirow[t]{2}{*}{ No pobre } & $0.1367 * * *$ & $0.1372 * * *$ & $0.1383^{* * *}$ & $0.1272 * * *$ & $0.1241 * * *$ \\
\hline & $-0,03$ & $-0,03$ & $(0.02691)$ & $(0.02666)$ & $(0.0265)$ \\
\hline \multirow[t]{2}{*}{ Ingresos laborales } & $-8,22 \mathrm{E}-09$ & $-8,37 \mathrm{E}-09$ & $-8,47 \mathrm{E}-09$ & $-5,60 \mathrm{E}-09$ & $-4,91 \mathrm{E}-09$ \\
\hline & $(0.00000)$ & $(0.00000)$ & $(0.00000)$ & $(0.00000)$ & $(0.00000)$ \\
\hline \multirow[t]{2}{*}{ desocupado } & $0.0751489 *$ & $0.0752832^{*}$ & $0.0749856^{*}$ & $0.0874841^{*}$ & $0.0857713^{*}$ \\
\hline & $(0.02531)$ & $(0.02522)$ & $(0.02527)$ & $(0.02082)$ & (0.02087) \\
\hline \multirow[t]{2}{*}{ edad } & $0.0019495^{* *}$ & $0.0018815^{* *}$ & $0.001895^{* *}$ & $0.0021355^{* *}$ & $0.0021141^{* *}$ \\
\hline & $(0.00082)$ & $(0.00086)$ & $(0.00086)$ & $(0.00086)$ & $(0.00085)$ \\
\hline \multirow[t]{2}{*}{ casado } & & $-0,0057425$ & $-0,0033425$ & $-0,0029566$ & $-0,003645$ \\
\hline & & $(0.02248)$ & $(0.02278)$ & $(0.02153)$ & $(0.02122)$ \\
\hline \multirow[t]{2}{*}{ femenino } & & & $-0,0118284$ & $-0,0035995$ & $-0,0050626$ \\
\hline & & & $(0.02141)$ & $(0.02066)$ & $(0.02045)$ \\
\hline \multirow[t]{2}{*}{ educación } & & & & $-0,0016439$ & $-0,0012463$ \\
\hline & & & & $(0.00277)$ & $(0.00277)$ \\
\hline \multirow[t]{2}{*}{ mestizo } & & & & & $-0,0502395$ \\
\hline & & & & & $(0.02704)$ \\
\hline \multicolumn{6}{|l|}{ Breusch Pagan } \\
\hline Chi2 (1) & & & & \multirow[t]{2}{*}{ Test: $\operatorname{Var}(\mathrm{u})=0$} & 1,93 \\
\hline P-Valor & & & & & 0,1644 \\
\hline Observations & 4797 & 4797 & 4797 & 4797 & 4797 \\
\hline$R^{\wedge} 2$ & $8,95 \%$ & $8,96 \%$ & $9,02 \%$ & $9,88 \%$ & $10,35 \%$ \\
\hline Individuos & 661 & 661 & 661 & 657 & 657 \\
\hline
\end{tabular}


Los datos indican que de manera estadísticamente significativa, las personas que manifiestan bienestar con una condición de no pobreza se declaran un $13 \%$ más felices que aquellos que no lo hacen. Este resultado es prácticamente invariable ante diferentes controles como ser casado, años de educación, estar ocupado, pertenecer al género femenino o pertenecer a la etnia mestiza. Así mismo, los datos reafirman que el salario no es una variable relevante por sí misma a la hora de determinar la felicidad en los individuos, como sí lo son la edad y la situación laboral.

Para analizar estos datos en contexto, se encuentra que el $24,14 \%$ de los individuos pertenecientes a la muestra declarados como no pobres, manifiestan en un $94,6 \%$ ser felices, mientras que el 73,86\% que dicen ser pobres manifiestan tan solo en un $80,8 \%$ ser felices. Así mismo, se encuentra que apenas el $5 \%$ de la población cuenta con salarios mensuales superiores a $\$ 2.400 .000$, mientras que el $50 \%$ recibe salarios mensuales inferiores a los $\$ 515.000$.

Los resultados en relación con las otras variables como sexo, educación, etnia y estado civil, además de no reportar significancia estadística, contribuye a la ambigüedad y variabilidad de resultados en trabajos empíricos en este campo tanto en contextos nacional, latinoamericano e internacional.

Este trabajo pretendió constituirse en una aproximación al tipo de variables relevantes que explican el grado de satisfacción o felicidad de los individuos a partir de sus condiciones de bienestar. En general, los resultados no fueron contrarios a la evidencia nacional e internacional. Se constató mediante regresiones econométricas simples de Mínimos Cuadrados Ordinarios (MCO) la relación positiva entre la felicidad de los países y su Índice de Desarrollo Humano (IDH). Es decir, por cada punto porcentual que aumente el IDH la felicidad lo hace en 15,6 puntos aproximadamente.

Este resultado es robusto a diferentes controles. Así mismo, la relación entre felicidad y PÍB per cápita se muestra marginal decreciente, ratificando la existencia de la Paradoja de Easterlin con un punto de inflexión en Usd\$ $21.815,6$ a partir de los cuales, incrementos de ingresos en los países generan una reducción del nivel de felicidad de los mismos.

Los datos también revelaron de manera significativa estadísticamente, que las personas que manifiestan bienestar con una condición de no pobreza se declaran un $13 \%$ más felices que aquellos que no lo son. Este resultado es prácticamente invariable ante diferentes controles como ser casado, años de educación, estar ocupado, pertenecer al género femenino o pertenecer a la etnia mestiza. Así mismo, los datos reafirman que el salario no es una variable relevante por sí misma a la hora de determinar la felicidad en los individuos, como sí lo son la edad y la situación laboral.

Finalmente, como dice Giarrizo ${ }_{1}$ es muy poco lo que se conoce aún sobre la felicidad. Conviven teorías, métodos y creencias sobre cuál es el camino que lleva al hombre a encontrarla. Se intenta entenderla y descubrirla permanentemente. Pero en esa búsqueda, lo poco que se sabe es que la felicidad es uno de los objetivos finales de la vida. Lo demás, es un proceso constante de búsqueda y satisfacción, de intentos a veces en vano por alcanzarla, y la economía no es ajena a ello, es una de las tantas llaves que tiene la cerradura del reino de la felicidad.

Una familia o una sociedad que tenga cubiertas un conjunto de necesidades importantes (algo más que las necesidades básicas) contará con un espectro de posibilidades mucho más amplio para buscar su camino. Así, un buen sistema económico no es aquel en el cual más cantidad de personas declaren ser felices, sino aquel que permita a su población más oportunidades para buscar esa felicidad. No existe una ciencia especialista en felicidad, el estudio de la felicidad no tiene fronteras disciplinarias, tiene aristas múltiples, y la economía es solo una de ellas.

El estudio permite concluir que este modelo encuentra la relación entre felicidad y bienestar 
en el municipio de Pasto, Colombia para el periodo de estudio. Se ratifica que las personas con mayor nivel de bienestar se declaran mayoritariamente felices en contraste con aquellos que según la evaluación de su propia vida, no lo han sido.

\section{Agradecimientos}

Se agradece de manera especial a las estudiantes Claudia Bastidas y Camila Paz. El autor declara que no tiene ningún conflicto de intereses

Conflicto de intereses: Ninguno declarado por los autores.

\section{Referencias}

1. Giarrizzo V. Economía y felicidad: ¿Existe vínculo? Buenos Aires. FCE/UBA, 2009.

2. CEDRE - Centro de Estudios de Desarrollo Regional y Empresarial. Cuentas económicas del municipio de Pasto. 2005 - 2010. San Juan de Pasto. Facultad de Ciencias Económicas y Administrativas, Universidad de Nariño, 2011.

3. ORMET. Red de Observatorios del Mercado de Trabajo. Diagnóstico socioeconómico y del mercado de trabajo Ciudad de Pasto. San Juan de Pasto. PNUD Oficina Colombia, 2012.

4. Andrews P, Withey S. Social indicators of Well-Being: American Perceptions of Quality, New York: Plenum Press, 1976.

5. Campbell A. The sense of well-being in America. New York. McGraw Hill, 1981.

6. Jahoda M. Current concept of positive mental. New York. Basic Books, 1958.

7. Gurin G, Veroff L, Feld S. Americans view their mental health: A nation wide interview survey. New York. Basic Books, 1960.

8. Bradum NM. The structure of psychological well-being. Chicago. Aldine Publishing. 1969.

9. Ware Jr, LE. The SF-36 Health survey, En: B.Spi1ker (ed.), Quality of life and pharmaco-economics in clinical trials. Philadelphia. Leppincott-Raven Publishers, 1996:337-45.

10. Van Praag B. The perception of welfare inequality. European Economic Review. 1997;10(2).

11. Easterlin R. Does economic growth improve human lot? Some empirical evidence, En: P.A. Davis, M.W. Reder, (eds.) Nations and households in economic growth. New York - London, Academic Press. 1974.

12. Scitovsky T. The Joyless economy. New York. Oxford University Press. Traducción al español: Frustraciones de la riqueza. México. Fondo de Cultura Económica (1986), 1976.

13. Layard R. Human satisfaction and public policy, the Economic Journal. 1980;90(360).
14. Veenhoven R. Happiness in nations: Subjective appreciation or life in 56 nations 1946-1992. Rotterdam. Erasmus University Press. 1993.

15. Oswald AJ. Happiness and economic performance. Economic Journal, Royal Economic Society. 1997;107(445):1815-31.

16. Frey BS, Stutzer A. Happiness and economics. Princeton University Press. 2002.

17. Kahneman D, Diener E, Schwartz N (eds). Well being: New York. The foundation of hedonic psychology. Russell Sage. 1998.

18. Freeman R. Job satisfaction as an economic variable. The American Economic Review. 1978;68(2).

19. Hamermesh D. Economics aspects of job satisfaction. Essays in labour market analysis. John Wiley \& Sons. 1977.

20. Ansa E, Miren M. Economía y felicidad: Acerca de la relación entre bienestar material y bienestar subjetivo. XI Jornadas de Economía Crítica Ecocri. España. 2008.

21. Bruni L, Porta P. Economics and happiness. Framing the analysis. Oxford University Press. 2005:7.

22. Ramírez-Gallegos R. La felicidad como medida del buen vivir en Ecuador: Entre la materialidad y la subjetividad. Ecuador. FLACSO, 2007.

23. Argyle M. La psicología de la felicidad. Madrid: Alianza Editorial. 1987.

24. Poveda-Bermúdez OM. Una revisión a los determinantes de la felicidad en América. Bogotá. Universidad Militar Nueva Granada. 2013.

25. Gianneti E. Felicidad, Barcelona: Paidos. 2006.

26. Silva-Colmenares J. Felicidad: La evolución como categoría científica y la relación con el desarrollo. Revista de la Información Básica CANDANE. 2008;3(1):62-77.

27. Pena-López JA, Sánchez-Santos JM, Iglesias-Vázquez E. Bienestar subjetivo, renta y bienes relacionales: Los determinantes de la felicidad en España. Revista Internacional de Sociología. 2013;71(3).

28. Frey BS, Stutzer A. Happiness, economy and institutions. The Economic Journal. 2000;110(466):918-938.

29. Cruz J, Torres J. ¿De qué depende la satisfacción subjetiva de los colombianos?. Cuadernos de Economía. 2006; XXV(45):131-154.

30. Happy Planet Index. The Happy Planet Index: 2012 Report. a global index of sustainable well-being.

31. WHOQOL Group. Study protocol for the World Health Organization project to develop a quality of life assessment instrument. Quality of Life Research Group. World Health Organization Quality of Life Instruments. 2005.

32. Clark A, Frijters P, Shields M. Relative income, happiness and utility: an explanation for the Easterlin paradox and other puzzles. Journal of Economic Literature. 2008;46:91-144.

33. Duesenberry JS. Income saving and the theory of consumer behavior. Cambridge, MA. Harvard University Press. 1949.

34. Esteve F. La economía de la felicidad. Nuevos elementos para la crítica del liberalismo económico. Filosofía y Economía de nuestro tiempo: orden económico y 
cambio social. Madrid. Ministerio de Educación y Ciencia. 2004.

35. Diener E, Scollon R, Napa Ch. Beyond the hedonic treadmill: Revising the adaptation theory of well-being. Dordrech. The science of well-being: The collected works of Ed Diener, edited by Ed Diener. Springer. 2009:103-118.

36. Easterlin R. Income and happiness: towards a unified theory. En: The Economics Journal, 111. 2001.

37. Layard R. Happiness: Has social science a clue? three lectures. First lecture: what is happiness?; Second lecture: Income and happiness: Rethinking economic policy; third lecture: What would make a happier society. Lionel Robbins Memorial Lectures. London School of Economic. Londres. 2003.

38. Layard R. La felicidad : lecciones de una nueva ciencia. Madrid. Taurus. 2005.

39. Veenhoven R. The four qualities of life. Ordering concepts and measures of the good life. Journal of Happiness Studies. 2000;1:1-39.

40. Veenhoven R. What we know about happiness. Working paper. Erasmus University Press. Rotterdam; 2001.

41. Cruz J, Torres J. ¿De qué depende la satisfacción subjetiva de los colombianos?. Cuad. Econ. 2006;25(45). 